\title{
Defining Burnout as a Dichotomous Variable
}

To the Editor:-In the July 2008 issue, Legassie et al. ${ }^{1}$ define burnout as having a high score in all three burnout domains of the Maslach burnout inventory (MBI): emotional exhaustion, depersonalization, and low personal accomplishment. Although the MBI is the gold standard tool for measuring burnout, there is considerable variability in how researchers studying physicians and medical students have defined burnout. For example, others have considered those with either high emotional exhaustion or high depersonalization as experiencing burnout. ${ }^{2}$ The effect of these differing definitions is significant. Legassie et al. report an overall burnout rate of $12.5 \%$. This grossly underestimates the burnout rate under alternative definitions, since at least 29\% of participants experienced high levels of either emotional exhaustion or depersonalization in the Legassie study.

Ultimately, burnout is a complex, continuous, and heterogeneous construct ${ }^{3}$ that manifests itself differently in different individuals. Emotional exhaustion, depersonalization, and inefficacy are symptoms of the syndrome. These symptoms can manifest in differing degrees resulting in burnout being best considered a continuum rather than a dichotomous variable. As advocated by Maslach, ${ }^{3}$ when evaluating the relationship between symptoms of burnout and other outcomes the ideal approach is to use the individual domain scores as continuous data. The second best option is to report categorized results separately using established definitions of low, average, and high cut-off scores ${ }^{3}$ for each domain. Using an overall burnout assessment (i.e. a dichotomous variable) is practical in research studies but problematic as there is no accepted standard definition. High scores on either the emotional exhaustion or depersonalization scales but not a low score on the personal accomplishment scale can distinguish the clinically burned out from the non-burned out, ${ }^{4}$ supporting the

Published online January 6, 2009 high emotional exhaustion and/or high depersonalization definition cited above. ${ }^{2}$ Another approach, supported by Maslach, is that an individual may be considered clinically burned out when a high emotional exhaustion score is present in combination with either a high depersonalization score or a low personal accomplishment score (Maslach, personal communication, September 24, 2008). Either approach is associated with trade-offs with respect to sensitivity and specificity. We note, however, that requiring high scores in all three domains is unsubstantiated by existing literature on the MBI.

We applaud Legassie et al.'s efforts to better understand physician well-being. Future research should incorporate consistent approaches for defining burnout using the MBI, rather than use variable definitions of burnout that prevent comparison across studies and slow efforts to identify, understand, and reduce burnout.

Liselotte N. Dyrbye, MD, Colin P. West, MD, PhD, and Tait D. Shanafelt, MD, Mayo Clinic College of Medicine, 200 First Street SW, Rochester, MN 55906, USA; (e-mail: dyrbye.liselotte@ mayo.edu).

\section{REFERENCES}

1. Legassie J, Zibrowski E, Goldszmidt M. Measuring resident well-being: Impostorism and burnout syndrome in residency. JGIM. 2008;23:1090-4.

2. Thomas NK. Resident burnout. JAMA. 2004;292:2880-9.

3. Maslach C, Jackson SE, Leiter MP. Maslach Burnout Inventory Manual. 3 ed. Palo Alto, CA: Consulting Psychologists Press; 1996.

4. Schaufeli W, Bakker A, Hoogduin K, et al. On the clinical validity of the Maslach burnout inventory and the burnout measure. Psychol Health. 2001;16:565-82.

$\mathrm{J}$ Gen Intern Med 24(3):440

DOI: $10.1007 /$ s11606-008-0876-6

(C) Society of General Internal Medicine 2008 